\title{
Intrapartum ultrasound - an integrated approach for best prognosis
}

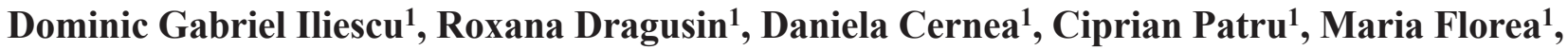 \\ Stefania Tudorache ${ }^{1}$
}

${ }^{1}$ Department of Obstetrics and Gynecology, University of Medicine and Pharmacy Craiova, Romania
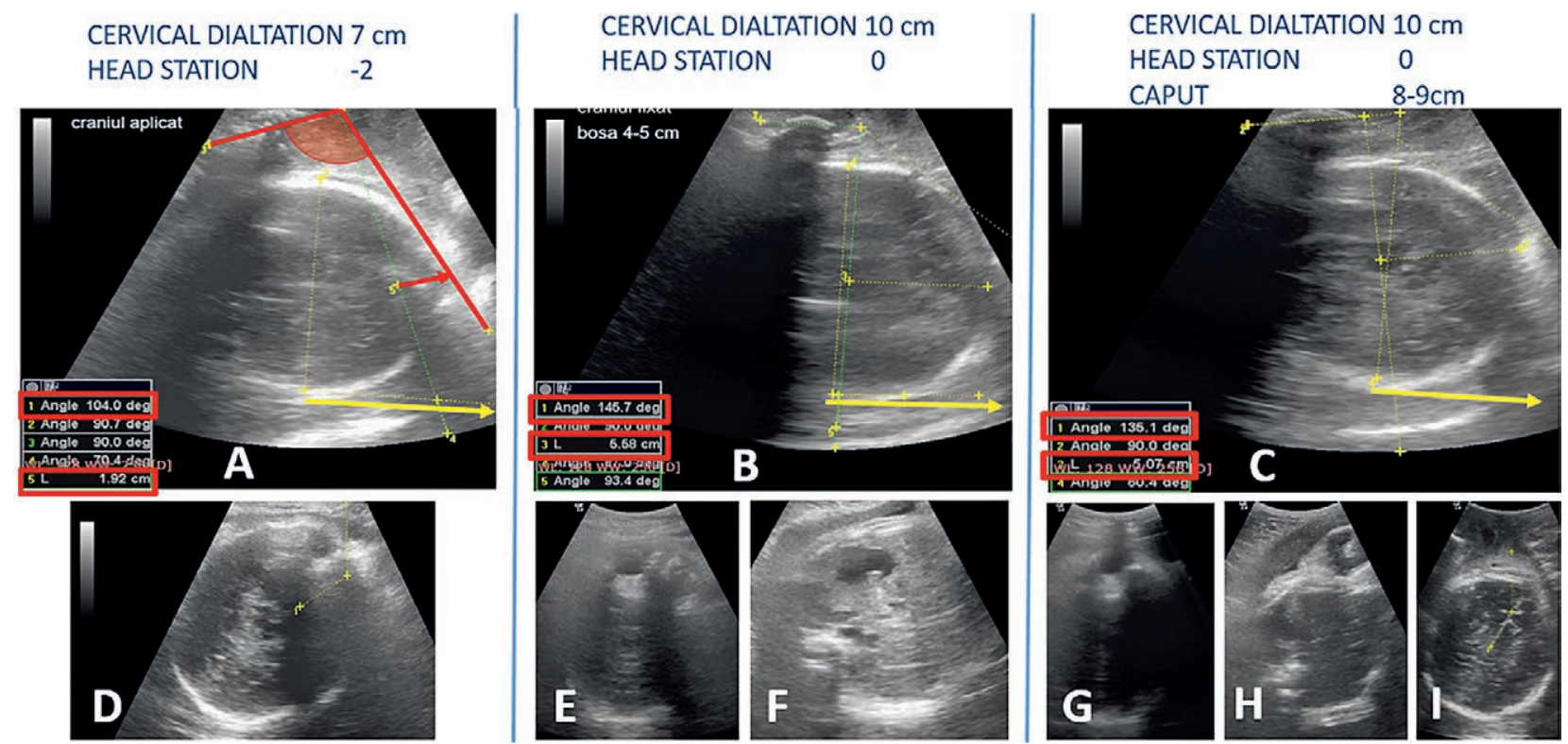

Fig 1. Evaluation of the fetal head progression and position during labor. The evolution of the fetal head progression parameters in transperineal sagittal views: progression angle and distance of $104^{\circ}$ and $1.9 \mathrm{~cm}(\mathrm{~A})$, increased above the cut-offs for vaginal delivery in early second stage of labor to $145^{\circ}$ and $5.6 \mathrm{~cm}$, respectively (B), suggesting successful head engagement. One hour later, stationary angle of progression- $135^{\circ}$ and progression distance- $5.1 \mathrm{~cm}(\mathrm{C})$. The evaluation of the fetal head position during labor shows persistent occipito-posterior position during labor in the first (D) and second stage (E,G) of labor in suprapubian transversal view, associated with persistent posterior position of the fetal spine in transabdominal axial view $(\mathrm{F}, \mathrm{H})$. Measurement of the midline angle in the transperineal subpubic transversal plane, shows persistent occiput-posterior position in the late second stage of labor and lack of head rotation (I). In the transperineal sagittal views, the persistent downward direction of the fetal head (A-C) and development of caput (C) is evident.

\section{To the Editor,}

In the last decade, several ultrasound (US) parameters were reported with high predictions regarding success-

Received 15.12.2016 Accepted 19.12.2016

Med Ultrason

2017, Vol. 19, No 1, 121-122, DOI: 10.11152/mu-932

Corresponding author: Roxana Dragusin

2 Petru Rares

200349 Craiova, Dolj, Romania

Phone: 0040769251576, Fax: 0040251502179

Email: roxy_dimieru@yahoo.com ful vaginal birth [1-3]. The case presented here reflects a situation, quite frequent in general practice, where the improper use of US measurements may diminish the confidence of labor care providers in the US data.

A low-risk laboring primipara was evaluated clinically and US, as part of an observational study aimed to determine the potential benefit of routinely intrapartum US monitoring (ClinicalTrials.govRegistry: NCT02326077). A favorable prognosis for vaginal birth was evident at the beginning of the second stage of labor based on the evolution of head progression parameters (fig $1 \mathrm{~A}, \mathrm{~B}$ ). However, 
the following clinical and US estimations (fig 1C) revealed an arrested labor, finalized with cesarean section delivery.

The outcome of this case is easily explained if we take into consideration an important aspect of the labor mechanism - the fetal head position. The US evaluations show a persistent occipito-posterior (OP) position (fig 1D-I), with posterior fetal spine, and "head-down" direction during advanced labor. A miss-interpreted favorable head progression may occur in this clinical situation because of the fetal head elongation and consecutively artificially increase of progression angle and distance measurements. This specific case was evaluated by a skilled operator who was aware of all these aspects. However, during our training and audit work in the field, we did encounter the examiners bias to estimate the prognosis of vaginal birth in the second stage of labor exclusively based on the head progression US measurements. Such situations may lead to disappointing results.

A recent study have stressed on the differences in the mechanism of labor between occipito-anterior and posterior positions [4]. The message of our letter underlines that the use of US is not meant to change the classic algorithm of labor monitoring. Thus, promising values of the fetal head progression parameters do not warrant vaginal delivery, and should be interpreted in the context of the head position. An important application of head progres- sion cut-offs was proposed for prolonged second stage of labor, but we should keep in mind that such clinical situations are frequently associated with OP position.

\section{Acknowledgements:}

This paper was published under the frame of European Social Found, Human Resources Development Operational Programme 2007-2013, project no. POSDRU/159/1.5/136893.

\section{References}

1. Molina FS, Nicolaides KH. Ultrasound in labor and delivery. Fetal Diagn Ther 2010;27:61-67.

2. Torkildsen EA, Salvesen KÅ, Eggebø TM. Prediction of delivery mode with transperineal ultrasound in women with prolonged first stage of labor. Ultrasound Obstet Gynecol 2011;37:702-708

3. Youssef A, Maroni E, Ragusa A, et al. Fetal head-symphysis distance: a simple and reliable ultrasound index of fetal head station in labor. Ultrasound Obstet Gynecol 2013;41:419-424.

4. Ghi T, Maroni E, Youssef A, Morselli-Labate A et al. Sonographic pattern of fetal head descent: relationship with duration of active second stage of labor and occiput position at delivery. Ultrasound Obstet Gynecol 2014;44:82-89.

\title{
A rare mechanical prosthetic valve dysfunction
}

\author{
Yan Sun ${ }^{1}$, Hui Ming Zhang², Hao Wang ${ }^{1}$
}

${ }^{1}$ State Key Laboratory of Cardiovascular Disease, ${ }^{2}$ State Key Laboratory of Cardiovascular Disease, Fuwai Hospital, National center for Cardiovascular Diseases, Chinese Academy of Medical Sciences and Peking Union Medical College, Beijing, People's Republic of China

\section{To the Editor,}

We describe the case of a 37-year-old woman with a long history of rheumatic multivalvular disease and an

Received Accepted

Med Ultrason

2017, Vol. 19, No 1, 122-123, DOI: 10.11152/mu-907

Corresponding author: Yan Sun

State Key Laboratory of Cardiovascular Disease Beijing, 100037, People's Republic of China

Phone: 008615110236678, Fax: 00861068331836

E-mail: sunxiaoyan01@sina.com operation for two mechanical valve prostheses in aortic and mitral positions 8 years prior presenation. Due to acoustic shadowing and artifacts, complete prosthetic valve evaluation by transthoracic echocardiography (TTE) was difficult and transesophageal echocardiography (TEE) was performed. The TEE showed that the mitral position was a single-disc valve with dysfunction. M-color Doppler showed the opening of the valve every two cardiac cycles. We also found the opening time of the mechanical valve in aortic positions was alternately long and short (fig 1). 


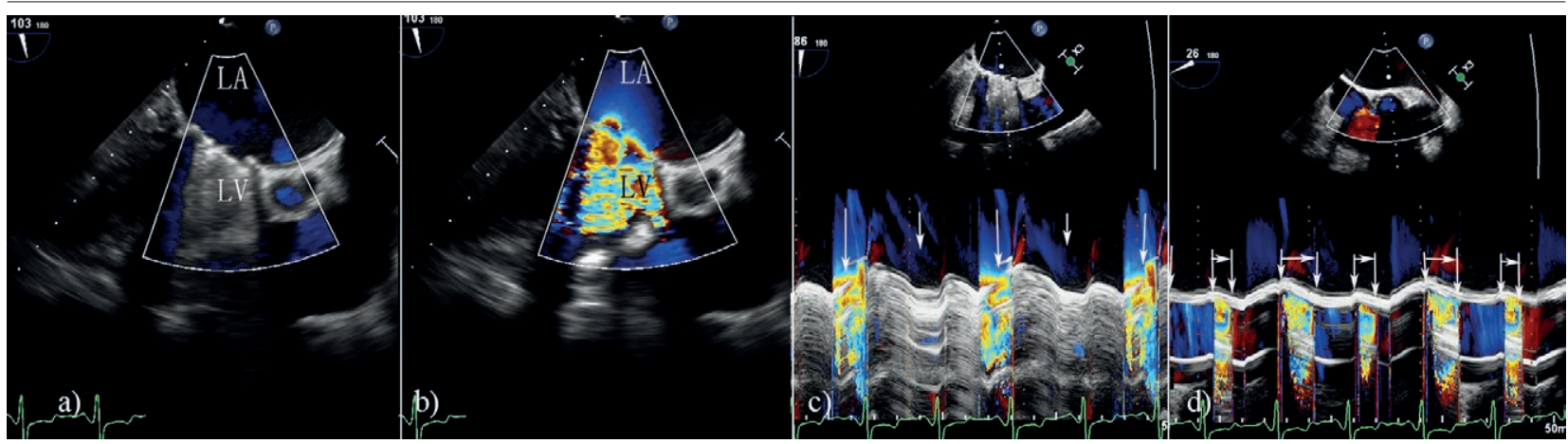

Fig 1. a) TEE: 2 -chamber view at $103^{\circ}$, with zoom in the LV inflow tract, color Doppler showing the single-disc valve movement. The mechanical valve closed during ventricular diastole period. LA: Left atrium; LV: Left ventricle; b) TEE: The same view and same period of cardiac cycles with fig 1a. The mechanical valve opened during ventricular diastole period. LA: Left atrium; LV: Left ventricle; c) TEE: 2-chamber view at $103^{\circ}$, with zoom in the LV inflow tract, M- Color Doppler. The mechanical valve opened once during two cardiac cycles. Long arrows indicate each time the valve open, short arrows indicate each time the valve not open during ventricular diastole period; d) Short axis view of large artery view at $26^{\circ}$, M-color Doppler, opening time of the mechanical valve in aortic positions was long and short alternately. Long arrows indicate each time the valve open, short arrows indicate each time that the valve do not open during ventricular diastole period.

Mechanical prosthetic valve dysfunction caused by pannus or thrombosis is an unusual but serious complication of heart valve replacement [1]. The most common symptom of prosthetic valve dysfunction is dyspnea, which may occur as a manifestation of obstruction in valves implanted both in aortic and in mitral valve position. TTE Doppler echocardiography is the method of choice for the evaluation of prosthetic valve function. In these cases that prosthetic valve dysfunction is suspected and TEE may be used. It is an accurate noninvasive method for assessing prosthetic valve function. Real time tridimensional TEE provides unique visualization and better understanding of the relationship between cardiac structures than two-dimensional imaging as well as accurate measurements valvular and ventricular functions [2].

\section{Reference}

1. Aoyagi S, Nishimi M, Kawano H, et al. Obstruction of St Jude Medical valves in the aortic position: significance of a combination of cineradiography and echocardiography. J Thorac Cardiovasc Surg 2000;120:142-147.

2. Lang RM, Mor-Aci V, Sugeng L, Nieman PS, Sahn DJ. Three-dimensional echocardiography: the benefits of the additional dimension. J Am Coll Cardiol 2006;48:20532069 